\title{
The Application of Folding Structure in Paper Packaging Design
}

\author{
Xiaoyi Zhang ${ }^{1, *}$ \\ ${ }^{1}$ College of Fine Arts, Soochow University, Suzhou, Jiangsu 215123, China \\ ${ }^{*}$ Corresponding author. Email: dhlg2011@163.com
}

\begin{abstract}
In the paper packaging structure design process, simply using the folding method can shape and fix the threedimensional structure of the paper packaging. Due to the simple process of pure folding carton, it has gradually replaced the traditional glued carton in many fields. However, due to the large amount of materials used in pure folding paper packaging, it will increase the cost of expenditure, so there are certain limitations. If folding is the main method and supplemented by interlocking and pasting methods, the cost can be effectively controlled and more space for the three-dimensional structure design of the packaging can be expanded.
\end{abstract}

Keywords: Paper packaging, Folding structure, Innovative design.

\section{INTRODUCTION}

Most people have been exposed to paper folding in their early childhood, and folding with paper is a common thing. The use of folding structure to design paper packaging has many commonalities with paper folding, which is equivalent to creating and developing common areas that consumers are familiar with. Therefore, it is very difficult to make things that are accustomed to be fresh. With the improvement of market demand, the design of paper packaging structure is developing in a more diversified direction. In this process, it is full of refinement and perfection of various materials and technical links. Consumers' psychology of seeking innovation and oddness has prompted designers to be more active in the transformation of design styles and the application of new technologies. Art design has a huge impact on human life, and it is directly related to and determines the quality of human survival and spiritual life. Therefore, designers are often considered and emphasized that they should pay more attention to the service objects and conduct in-depth research on human material and spiritual needs in order to better serve

*Fund: Funding Project for the Construction of

Preponderant Disciplines in Jiangsu Colleges and Universities the human race. ${ }^{1}$ Using exaggerated shapes to form a strong visual impact will naturally attract people's attention, but if the structural design cannot stand up to careful scrutiny and speculation, people's attention will be short-lived. As the most familiar material to the public, in order to obtain continuous attention and recognition through the design of the structure, it must be carefully considered and arranged in many details. Only precise, considerate and intriguing designs can occupy people's hearts for a long time.

The folding structure is suitable to be moulded with simple and smooth lines, which can give people a refreshing and magnificent feeling, while the design works that are folded too much will give people a cumbersome and trivial impression. In the basic form of paper packaging, the folding lines are mostly straight lines. In the design of the folding structure of paper packaging, the folding lines can be changed into an arc, a slash, or a combination of arc and slash, so that the carton produced can present many unexpected looks and styles. If the arc or the combination of the arc and the slash is used, the finished carton will have a completely different three-dimensional shape when viewed from the front and the side, with a very unique three-dimensional shape and strong visual

1. Huang Lusha, Huang Xun: "The Application and Exploration of 'Deconstruction and Reconstruction' Thinking in Art Design", Art Work, Shenyang, Issue 5, 2018, p. 106. 
recognition. The moulding of the paper packaging can be completed by a single folding process, or supplemented by a combination of interlocking and pasting processes. The combination of different processes has different technological advantages, and the presented works are also different in thousands ways.

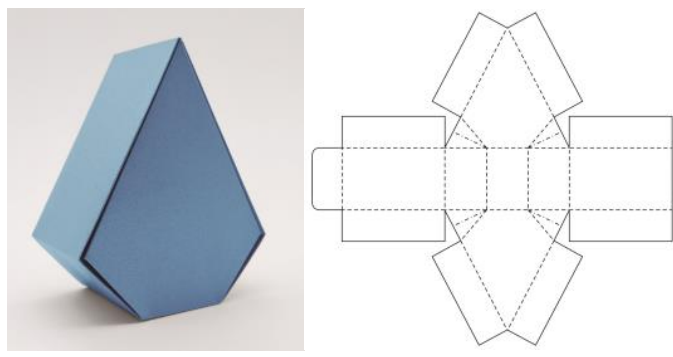

Figure 1 A pentagonal packaging box. ${ }^{2}$

\section{THE MOULDING THROUGH A SINGLE FOLDING PROCESS}

After the paper is folded, it can be made into various three-dimensional shapes. Most of the common polygonal packaging boxes on the market will use the cement process, however, the pentagonal packaging box of "Figure 1" has no glue and its moulding is completely through folding. The structure is designed ingeniously, rigorously and firmly, and the protection performance is good. Folded packaging mostly uses a tightening folding structure, which clenches and clamps the intersecting surfaces to each other, and adds friction between the papers to be a fixed moulding. The folding structure in "Figure 2" is naturally joined with the box body, providing the product with good cushioning performance and vibration absorption performance. Two packages of the same type can be combined to form a complete cuboid, which has the advantages of light weight, space saving, good protection, strong adaptability, material saving, and environmental protection and so on. If one wants to make a mature packaging structure work, one needs to accurately control every link, and then carefully compare and confirm the details. There will be many changes in the folding process. If it is not adjusted in time, even if it can be moulded, the degree of firmness and aesthetics will be greatly reduced.
2. The pictures quoted in this article are all made by the author herself.

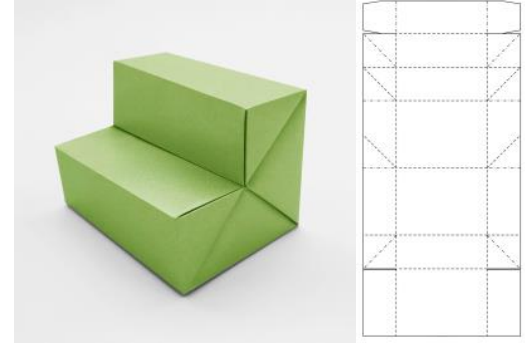

Figure 2 A moulded packaging box through folding.

\section{THE MOULDING THROUGH FOLDING AND SUPPLEMENTED BY INTERLOCKING PROCESS}

If the three-dimensional structure cannot be fixed only by folding, an interlocking structure can also be added to assist positioning, and the threedimensional shape can become more abundant. The " \# "-shaped box in "Figure 3" doesn't use an adhesive structure, but it cannot be firmly moulded by relying on the folding structure alone. The "\#"shaped structure of the bottom box needs to be supplemented by an interlocking structure to form a three-dimensional structure, and the four edges are plugged into each other up and down respectively. The interlocking process requires precise scale design, and the geometric structure of the socket needs to be tightly closed to resist compression and tension. The box body has a hollow double-layer structure on all four sides, and the interlocking structure is also a double-layer structure, which provides a strong guarantee for the firmness of the packaging structure. The bull head-shaped packaging box in "Figure 4" doesn't use an adhesive structure, either. The originally folded cover and the bottom box cannot be independently fixed and moulded. It is necessary to interlock the "bull ears" on the cover and the "horns" on the bottom box to each other to present the overall shape of the bull head. In order to ensure the firmness and stability of the interlocking structure, the facades where the interlocking structure is located are designed as a double-layer structure. After the production of the plan of the work is completed, a sample must be produced for comparison and confirmation in size. Because of the thickness of the paper, the size will change during the folding and interlocking process. If it is not adjusted in time, even if it can be moulded, the accuracy, firmness and aesthetics will be greatly reduced. 


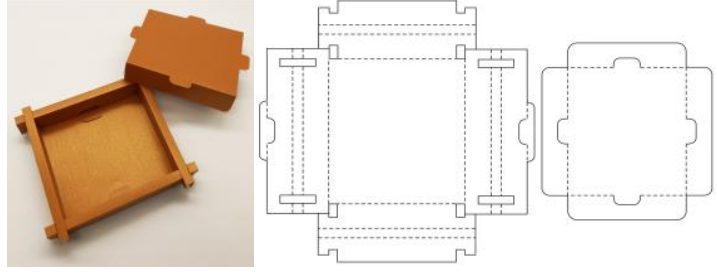

Figure 3 A "井"-shaped packaging box.

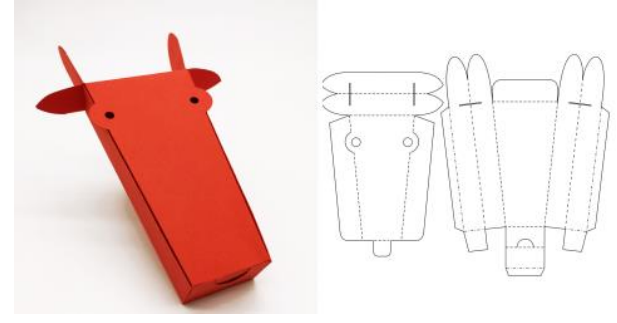

Figure $4 \mathrm{~A}$ bull head-shaped packaging box.

\section{THE MOULDING THROUGH FOLDING AND SUPPLEMENTED BY PASTING PROCESS}

Pure folding cartons also have certain limitations. From the perspective of cost, pure folding carton uses more paper, and the material cost of the pure folding structure of the same volume of carton container will be higher than that of the adhesive structure. Therefore, partial use of glue and folding of the main structure is also a common manufacturing process at present. The designer should select the processing method according to the different needs of the product.

The six-pointed star chocolate packaging in "Figure 5" adopts a cover and tray carton structure. The structure of the cover is folded to shape the top surface into a high and low double-layer threedimensional shape formed by 24 triangular sections, with a unique and novel shape, highlighting the three-dimensional beauty of the folding structure. The ridges connected by the six corners are fixed with a double-layer adhesive structure to enhance the firmness. The inner diameter of the carton cover of the cover and tray carton structure must be equal to the outer diameter of the bottom box plus the friction of the paper, so that it can be perfectly covered and not easy to fall off each other. The precise measure can effectively improve the firmness of the carton structure during transportation and storage, and effectively resist external forces.

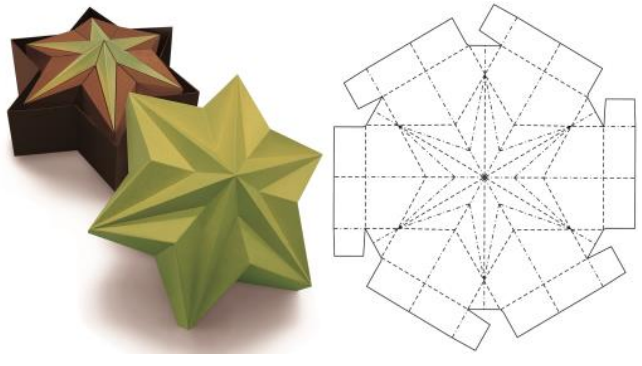

Figure 5 A chocolate packaging box.

Folding technology is most frequently used in the design of paper packaging structure. Simple folding methods have become commonplace, and it is difficult to attract consumers' interest. Therefore, it is necessary to make folding innovative and interesting in order to raise the interest of consumers. In addition to changing the shape of the line, that is, changing the straight line to the broken line and the broken line to the arc, as well as switching the direction of the broken line to fold inward and fold outward, one can also nest the folded structure. The tower-shaped packaging box in "Figure 6" is based on a four-sided triangular pyramid carton structure, and the carton is folded in a horizontal direction inside and outside alternately on the surface of the box body to fold the carton into a five-layer tower-shaped overlapping structure, which can be used as the packaging of products with special forms, and has a good display effect. In this packaging structure, the horizontal broken line distances of the inwardly nested parts of each layer are equal, but the horizontal heights of the outer five-layer tower-shaped structure are not equal. The sizes of the two are variable items. Designers can set different sizes according to product needs, and the finished effects presented by different sizes are also different. There is one rule in the design of nested cartons that must be followed, that is, the broken lines on the surface of the box body must be alternately folded inside and outside in a direction parallel to the bottom edge of the box. If the broken line and the bottom edge are not parallel, the surface of the carton is uneven and cannot be placed stably.

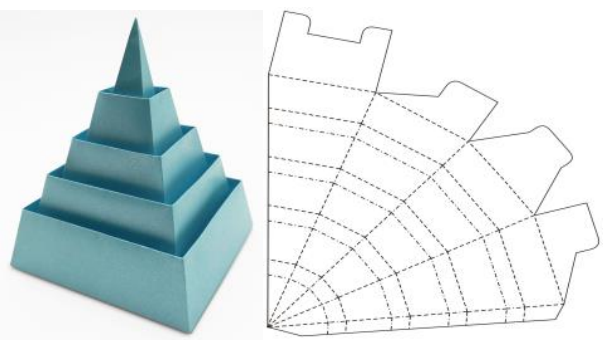

Figure 6 A tower-shaped packaging box. 
The folding structure on the paper packaging can realize many complex and unprecedented forms. Most of the common paper packaging structures on the market are symmetrical structures around the sides. Designers can also try to design pairwise symmetrical structures. For example, "Figure 7" is a pairwise symmetrical packaging box. Each of the opposite sides of the box, namely the front and the back, the left and right sides have the same symmetrical structure, but the two adjacent sides are different, so the threedimensional shapes presented by the front and side are completely different. The styles of using straight or curved lines when shaping the structure are also very different. The "Figure 8" Chinese women's wear packaging box uses an arc broken line to outline the female waist curve, which enhances the interestingness and imagination of the packaging box. The left and right sides of the box can be opened, and when the cover is opened, one can easily pick up and place items. It is moulded with a piece of paper and has only one pasting place. The design and production process is simple, the simulation image is vivid, and the cost factor is also considered while satisfying the visual effect. The arched arc structure can open and flatten the supporting structures on both sides to form a flat structure during transportation, which greatly saves transportation and storage space, satisfies the visual effect and excellently controls the cost and energy consumption, and avoids the problem of waste of space during transportation and storage of specialshaped cartons due to the one-sided focus on changeable shapes.
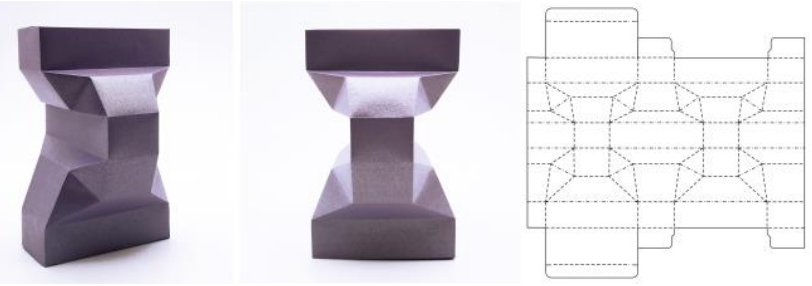

Figure 7 A pairwise symmetrical packaging box.

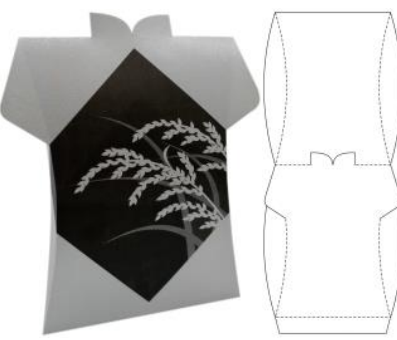

Figure 8 Chinese women's wear packaging box.

In addition to the pairwise symmetrical structure, designers can also try the convoluted structure, which also has a lot of creative expression space: When designing, designers can choose vertical convoluting or oblique convoluting by using broken line, curve folding or convoluted methods, and the finished three-dimensional effects are very different. The basis for dividing vertical convoluting and oblique convoluting here is: If each ridge of the packaging box has a convoluting tendency, it is regarded as vertical convoluting; however, if each ridge of the packaging box has a convoluting tendency and the line between the apex and the bottom end of the ridge is slant, it is regarded as oblique convoluting. Although the packaging box of "Figure 9" has many inclined broken lines on the surface, because the line connecting the top to the bottom of the ridge is vertical, the overall structure is still a vertical convoluting structure. The convoluting structure doesn't appear frequently in the market, and visually makes people feel that the process is more complicated, but in fact, there is no essential difference from the ordinary structure. If one can make a bold and reasonable design on the basis of the convoluting structure, it will have an effect of half the work with double results, form a unique visual charm, and easily win the favor of consumers. In "Figure 10", it changes the edges of the common flat shake-lid to a slightly slanted curve to have a unique and enchanting shape. Using these techniques to transform and change the common basic carton shapes will bring unknown surprises. It is true that earth-shaking changes can bring strong impacts, but small changes in life can bring inexplicable warm.
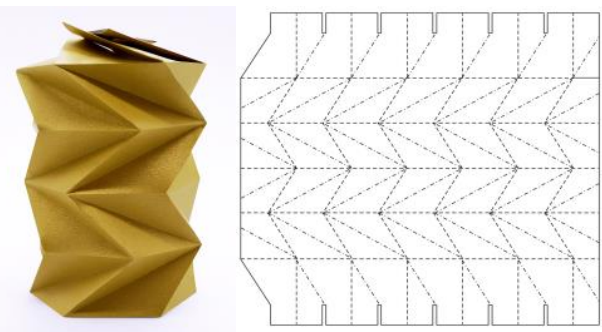

Figure 9 A packaging box of broken line vertical convoluting. 


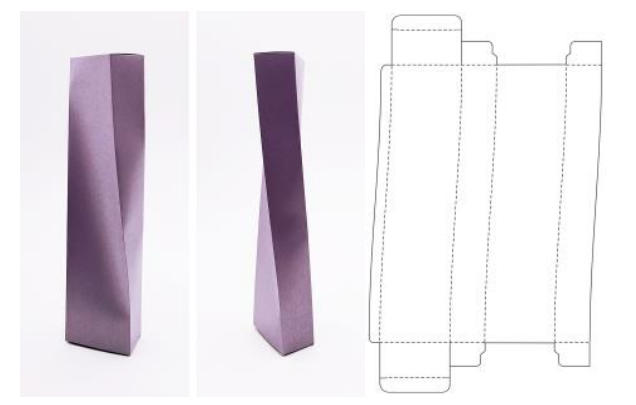

Figure 10

A packaging box of curve oblique convoluting.

\section{CONCLUSION}

Before the work of packaging structure design is carried out, designers should conduct design research on related products. For the design itself, the process of investigation and research can help it find new sources of inspiration, enrich its imagination, cultivate creative thinking, and can also help it learn new knowledge and information from related matters in the past. Through investigation and research, designers can obtain relevant information about the design direction and visual expression techniques. ${ }^{3}$ Regardless of whether one chooses a single folding process or supplemented by a combination of interlocking and pasting processes, one must pay attention to avoiding complicated processes and difficult assembly structures, removing unnecessary and cumbersome elements, and improving the packaging efficiency as much as possible. The packaging with reasonable structure and simple and fast use process can bring fresh and beautiful emotions to users. Designers need to scrutinize and analyze to find a breakthrough in the design of the structure. Each design requires further consideration and refinement of the previous concepts and methods before the design can be carried out. In the design process, attention should be paid to many factors such as cost, craft, practicability, and transportation convenience, and the form and function should be combined to make overall planning for the design. Only packaging structure works that meet the aesthetic and emotional needs of consumers can gain the longterm attention and recognition of consumers.

\section{AUTHORS' CONTRIBUTIONS}

This paper is independently completed by Xiaoyi Zhang.

\section{REFERENCES}

[1] Huang Lusha, Huang Xun: "The Application and Exploration of 'Deconstruction and Reconstruction' Thinking in Art Design", Art Work, Shenyang, Issue 5, 2018, p. 106. (in Chinese)

[2] Zong Cheng: "The Purpose and Service of Design Research", Art Work, Shenyang, Issue 5, 2017, p. 97. (in Chinese)

[3] Yang Zongkui: "Packaging Design", China Youth Publishing House, Beijing, 1998. (in Chinese)

[4] Kenya Hara: "Design of Design", Shandong People's Publishing House, Jinan, 2006. (in Chinese)

[5] Zhang Xiaoyi: "Paper Packaging Creative Design Tutorial", Jiangxi Fine Arts Publishing House, Nanchang, 2005. (in Chinese)

[6] Zhang Xiaoyi: "Paper Package Structure Creativity \& Design", Chemical Industry Press, Beijing, 2019. (in Chinese) 